\title{
Study of the dynamic Bauschinger effect in Ti6Al4V by torsion experiments
}

\author{
J. Peirs, P. Verleysen, and J. Degrieck \\ Ghent University, Department of Materials Science and Engineering, 9052 Zwijnaarde, Belgium
}

\begin{abstract}
The materials kinematic hardening behaviour and Bauschinger effect is indispensible to describe complex deformation processes involving strain path changes. Moreover, the Bauschinger effect provides valuable information about underlying microstructural plasticity mechanisms. Until now, the Bauschinger effect at high strain rates remains a largely unexplored topic. However, different studies demonstrated the strain rate dependent character of the Bauschinger effect. The aim of this work is to study the dynamic Bauschinger effect by means of a novel experimental technique. A modified torsional split Hopkinson bar setup is used to conduct Bauschinger experiments on Ti6Al4V. Forward and reverse loading of the specimen take place successively in only one experiment. This has the advantage of having the same thermal conditions during the two loading cycles. Besides high strain rate tests, quasi-static torsional Bauschinger experiments are conducted. The Bauschinger effect at the different strain rates is quantified with a dimensionless Bauschinger stress parameter. It is found that the Bauschinger effect is present at all tested strain rates. However, it is more pronounced at high strain rates. This implies that the kinematic hardening of Ti6Al4V is strain rate sensitive.
\end{abstract}

\section{Introduction}

The Bauschinger effect consists of the reduction of compressive yield stress of engineering materials after tensile cold work. In general, the material's strain hardening behaviour consists of an isotropic and a kinematic component. The isotropic hardening involves the symmetrical expansion of the yield surface while the kinematic hardening involves a translation of the yield surface. An accurate isotropic and kinematic hardening model is necessary for simulation of real forming processes in which strain path changes occur. In many applications materials are stretched and rapidly deformed into opposite directions during one production step. In addition kinematic hardening behaviour plays an essential role for FEM simulation of spring-back prediction [1]. Furthermore, the materials kinematic hardening provides valuable information about underlying microstructural plasticity mechanisms. Although from a different origin, the strain path is also important for materials that undergo twinning because twinning is sensitive to the sign of the load.

Usually, quasi-static tension-compression Bauschinger tests are used to assess the materials kinematic hardening behaviour. In such test, the sample is loaded consecutively in tension and in compression. The difference in the flow stress of the two load directions (Bauschinger effect) is a measurement of the materials kinematic hardening. Hereby, it is important to have the same specimen geometry and boundary conditions in the forward and reverse loading step, which is not easily achieved because the geometry changes during deformation. Therefore, Bauschinger tests in shear [2] have a distinct advantage because the cross section of the specimen remains constant. Torsional Bauschinger tests are a particular case of Bauschinger tests in shear with two major advantages compared to more traditional compression-tensile
Bauschinger tests. In addition to the fact that using the same specimen geometry in both loading cycles eliminates the structural specimen response from the results the same stress state is present during the forward and reverse loading cycle. In this way, the effects of the stress triaxiality and possible tensile/compression asymmetric material behaviour are eliminated.

Special experimental techniques have been developed to study the high strain rate behaviour of metals. However, the combination of high strain rate tests and strain path changes is rare in literature. In 1968, Nevill and Myers [3] conducted static tensile tests followed by dynamic compression tests on 1100 aluminium. Although the conclusion of this study was that the Bauschinger effect is independent on the strain rate, machining the specimen for the compression tests from the tensile specimen caused an elapsed time between loadings in the order of 48 hours. In 1976, Eleiche and Campbell [4] studied the effect of the strain rate during reverse torsional loading. They point out the importance of a short time between the forward and reverse loading to avoid any microstructural changes due to ageing or recovery. Their specimens were pre-loaded at low strain rate $\left(0.006 \mathrm{~s}^{-1}\right)$ and afterwards dynamically loaded in the opposite direction. Both the pre-loading and reverse loading were done with the same torsional Hopkinson setup to limit the time between the loadings to a few microseconds. They found that the Bauschinger effect becomes more pronounced at large pre-strains and high strain rates. More recently, Thakur and Nemat-Nasser [5] studied the Bauschinger effect in two aluminium alloys under dynamic loading conditions. They used an interrupted tensile test followed by a compression test. Also these tests showed that the Bauschinger effect is more pronounced at high strain rates. To our knowledge, no publications exist in which the dynamic forward and reverse loading steps follow each other within a millisecond. 


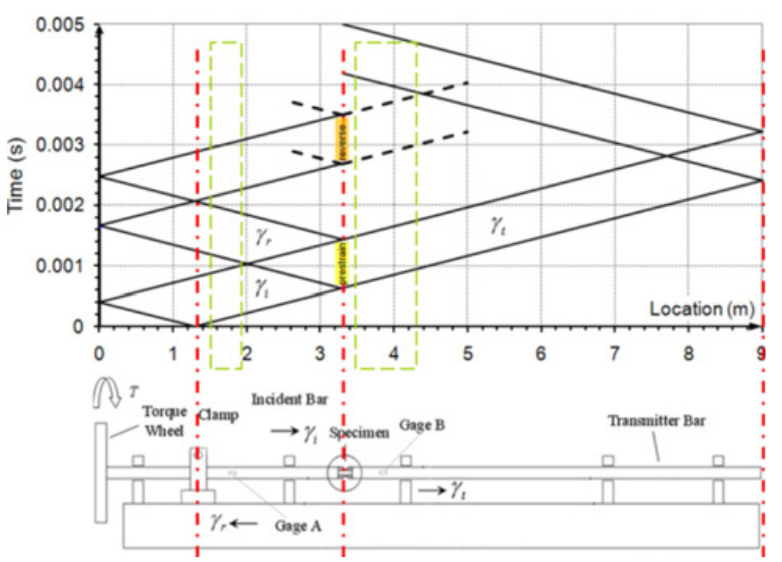

Fig. 1. Lagrange diagram and configuration of Hopkinson setup for Bauschinger tests.

\section{Experimental technique}

\subsection{Torsional split Hopkinson bar test}

The split Hopkinson bar technique is based on elastic wave propagation in slender bars. A small specimen is fixed between two Hopkinson bars. Thin-walled tubular specimens are used for torsion tests. Connection of the specimen with the Hopkinson bars is accomplished by hexagonal flanges and socket.

A torsional loading wave $\gamma_{i}$, generated in the input bar by suddenly releasing a pre-twisted section of the input bar, propagates along the bar towards the specimen. The length of the incident wave corresponds with two times the length of the pre-twisted section and the amplitude corresponds with half the applied torque. The specimen is dynamically loaded when the incident wave reaches the specimen [6]. Torsion samples are carefully machined from extruded bars of $16 \mathrm{~mm}$ diameter. The composition of the material is published in [7]. The inner diameter of the specimen is $9 \mathrm{~mm}$ and the length is $2 \mathrm{~mm}$. The wall thickness has a small variation of $0.42 \mathrm{~mm}$ at the sides to a minimum of $0.40 \mathrm{~mm}$ at the centre of the gauge section to be sure that strain localization and fracture occurs in the centre of the specimen.

\subsection{Torsional Bauschinger test}

In a Bauschinger test, the material is loaded two times in opposite direction (pre-strain and reverse strain). The Hopkinson setup needs a modification to accommodate these tests. In the classical setup the input bar has a larger length than the output bar which is the most efficient use of the bar-length with respect to maximal achievable specimen loading time. In the modified setup, the length of the output bar is increased to approximately two times the length of the input bar. The reason for this configuration is explained with the help of the Lagrange diagram shown in figure 1.

The location of the clamp and specimen is indicated with vertical dash-dot lines. The input and output bar have lengths of respectively $3.2 \mathrm{~m}$ and $5.8 \mathrm{~m}$. The incident wave $\gamma_{i}$, generated at time $=0$ by releasing the clamp,

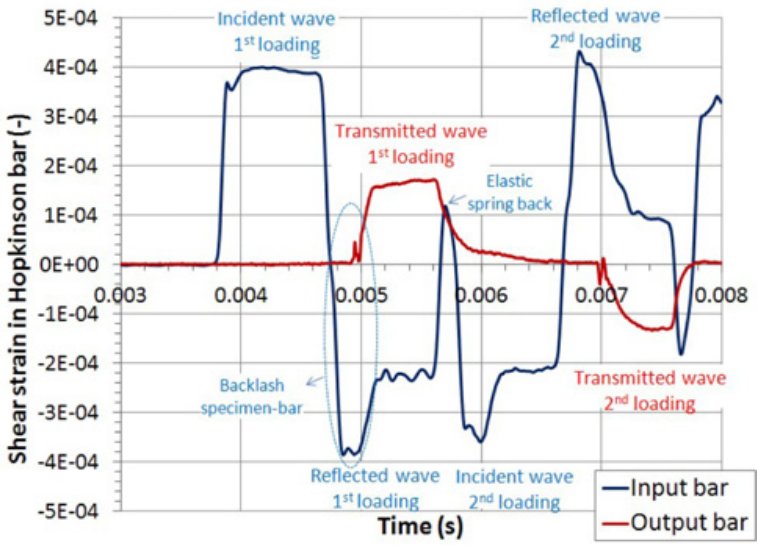

Fig. 2. Strain waves measured in the input and output bar. The waves have been time shifted to the location of the specimen.

propagates towards the specimen. As the wave interacts with the specimen a part, $\gamma_{t}$, is transmitted to the output bar and the remaining part, $\gamma_{r}$, is reflected into the input bar. The sign of the reflected wave is opposite to the sign of the incident wave. The reflected wave propagates away from the specimen and reflects a second time on the inertia disc at the left end of the input bar. In this second reflection, the sign of the wave remains unchanged. Thus, the resulting torsional wave has opposite sign to the incident wave $\gamma_{i}$. Consequently, this wave will load the specimen a second time but in the opposite direction as the first loading. There is no risk of interaction with the transmitted wave from the first loading because the output bar has a length greater than twice the input bar.

An example of strain waves actually measured during a torsional Bauschinger test is shown in figure 2. The effect of backlash in the specimen-bar connection and the elastic spring back of the specimen after the first loading can be observed on the figure. The incident wave is reflected completely as long as backlash exists. The advantage of the backlash is a high strain rate at the onset of specimen loading.

The strain that can be achieved in the reverse loading step is always lower than in the forward step. This is not dramatic because most information on the materials kinematic hardening is derived from the first percents of reverse plastic strain. The strain rate during the second loading is lower than for the first loading too, because the amplitude from the incident wave is lower. The effect of this small difference of the strain rate on the flow stress can be corrected afterwards, taking into account the strain rate dependent behaviour of the material.

The time between the two loading steps is around $1 \mathrm{~ms}$. This is short enough to neglect a possible temperature decrease of the specimen. However, on microscopic level this time can be enough to cool down local hot spots, affecting the formation of adiabatic shear bands.

Tuning the amount of pre-twist (strain rate) and the length of pre-twisted section of the loading bar (loading duration) is critical for a successful Bauschinger test. At the one hand the strain rate and loading time need to be high enough to produce plastic deformation in the reverse loading step. On the other hand, if the strain rate and 


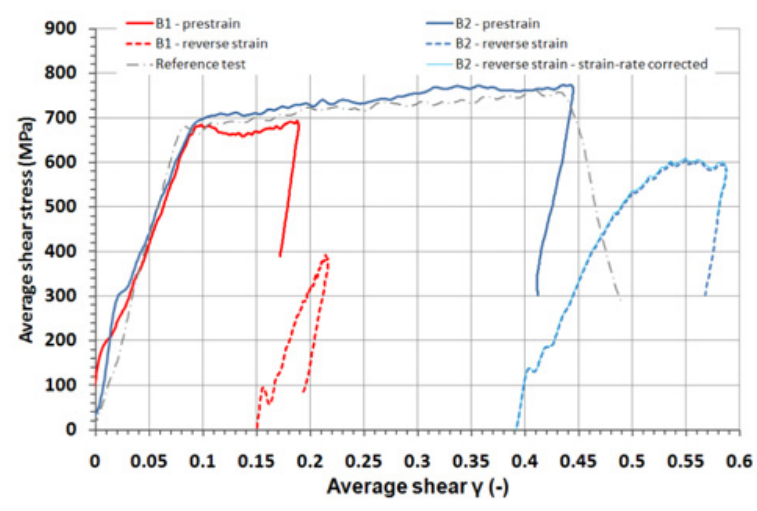

Fig. 3. Stress-strain curves of two Bauschinger tests.

loading time are too high the specimen will fail in the prestrain step. Furthermore, backlash between the hexagonal flange of the specimen and the hexagonal socket on the bar ends complicates the determination of right test settings and affects the accuracy of the experimental results due to misalignment of the specimen.

\subsection{Digital image correlation}

Since a Hopkinson test yields the force and displacement history of the specimen, only average values of the shear stress and strain in the specimen can be calculated. For assessing the local material behaviour, full-field strain measurements by digital image correlation (DIC) are performed. The deformation of a fine speckle pattern applied on the specimens' surface is recorded with a high speed camera. Then the images are corrected to take into account the curvature of the surface of the tubular specimen [8]. Finally, the images are processed with the non-commercial DIC software MatchID [9] to calculate the local strain distribution.

\section{Experimental results}

The results of two Bauschinger tests (B1 and B2) are presented in figure 3. In the first test B1, the amplitude of the incident torsion wave is too low to have a sufficiently high strain rate in the reverse loading step. Consequently, no plastic deformation is reached. In the second test $\mathrm{B} 2$, there is considerable reverse plastic deformation. The specimen is not broken at the end of the test. The yield stress during reverse loading is less well defined than in the forward loading stress strain curve. The yield stress is significantly lower than during the forward loading. The maximal shear stress in the reverse loading is $600 \mathrm{MPa}$ which is approximately $30 \%$ lower than the stress reached during the pre-strain. This test shows that Ti6Al4V exhibits an important Bauschinger effect at high strain rates.

The average strain rate during the pre-strain loading of test B2 is $\pm 700 \mathrm{~s}^{-1}$ against $\pm 300 \mathrm{~s}^{-1}$ in the reverse loading. The effect of the different strain rates on the flow stress is negligible as can be seen on the strain rate corrected curve. The Johnson-Cook model with the parameters that are identified in a previous paper [10] is used for this correction. Similar strain rate sensitivity during reverse shear is thereby assumed.

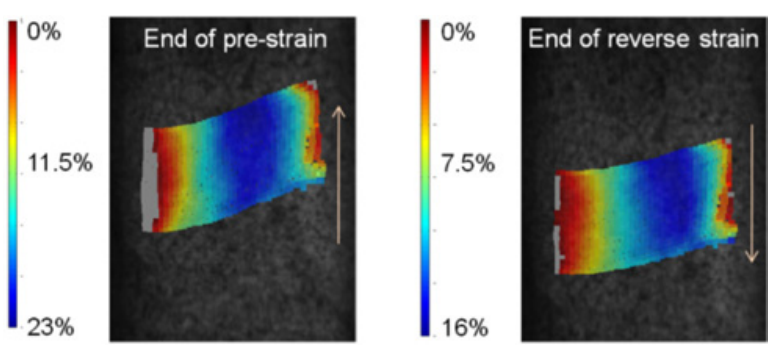

Fig. 4. Local strain measurement during Bauschinger test.

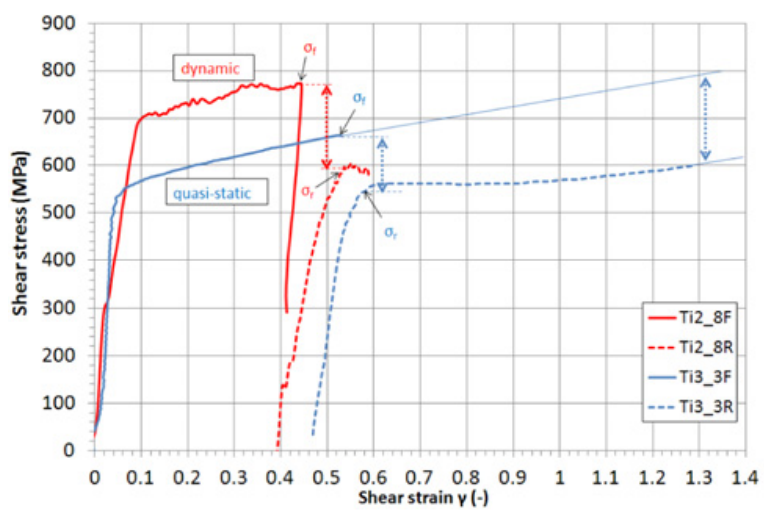

Fig. 5. Comparison between dynamic and quasi-static Bauschinger test.

Digital image correlation is used to assess the distribution and evolution of the local strain in the specimen. Two contour plots of the shear strain are shown in figure 4 . The image on the left hand side shows the strain distribution at the end of the pre-strain loading. It is noted that the zone where strain is measured had a rectangular shape in the undeformed specimen. On the right hand side of figure 4, the strain distribution at the end of the reverse strain step is represented.

An interesting feature is found in the high strain that is reached in B2 without fracture. The maximal shear strain measured with DIC in the pre-strain loading is $23 \%$ which corresponds more or less with the materials average fracture strain measured in other torsion tests. The maximum measured strain in the reverse loading is $7 \%$. One could expect the specimen to break after this high plastic deformation.

Besides the high strain rate Bauschinger test, a quasistatic Bauschinger experiment is carried out too. Figure 5 shows the corresponding stress-strain curves. Obviously, the Bauschinger effect is also present during quasi-static loading of Ti6Al4V. Next to the Bauschinger effect there is also a permanent softening of the material deformed in the opposite loading direction.

A so-called Bauschinger effect parameter $\beta_{\sigma}$ is introduced to quantify the Bauschinger effect [38]:

$$
\beta_{\sigma}=\frac{\sigma_{b}}{2 \sigma_{f}}=\frac{\sigma_{f}-\left|\sigma_{r}\right|}{2 \sigma_{f}}
$$

where $\sigma_{f}$ is the maximum flow stress in the forward loading direction and $\sigma_{r}$ the yield stress upon reloading in the reverse direction. The stress value $\sigma_{b}$ is known as the back stress. The smaller the value of $\beta_{\sigma}$, the smaller the Bauschinger effect. For the experiments presented in figure 5, the Bauschinger-effect parameter is 0.087 at 
low strain rates while it is 0.114 at high strain rates. This difference implies that the kinematic hardening of Ti6Al4V is strain rate sensitive.

\section{Conclusions}

An alternative configuration of the Hopkinson bars of a torsional Hopkinson setup enabled Bauschinger tests, i.e. experiments with reversal of the loading direction. The advantage of the presented method in comparison with already existing techniques is that loading in the two directions occurs within one single experiment. Consequently, the elapsed time between the two opposite loadings is short. The disadvantage of the method is that the strain that can be reached in the reverse loading direction is rather limited.

The experimental results show that the technique is promising for assessing the materials kinematic hardening behaviour. A very pronounced Bauschinger effect is found in dynamically loaded Ti6Al4V.

Apart from dynamic Bauschinger tests, quasi-static test has also been carried out. Comparing both results, it is found that the Bauschinger effect in Ti6Al4V is more pronounced at high strain rates than at quasi-static strain rate. It is concluded that the Bauschinger effect is strain rate dependent.

\section{Acknowledgements}

The authors would like to acknowledge funding of The Interuniversity Attraction Poles Program (IUAP) of the Federal
Science Policy of Belgium and the partners of IUAP-VI (www.m3phys.be). Furthermore, the authors acknowledge the technical support of Dr. P. Lava and Dr. D. Debruyne from the Catholic University College Ghent for their in-house developed image correlation software MatchID.

\section{References}

1. J.L. Alves, S. Bouvier, M. Jomaa, R. Billardon, L.F. Menezes, Materials Processing and Design, 908, 691 (2007)

2. S. Bouvier, H. Haddadi, P. Levee, C. Teodosiu, Journal of Materials Processing Technology, 172, 96 (2006)

3. G.E. Nevill, C.D. Myers, Journal of the Mechanics and Physics of Solids, 16, 187 (1968)

4. A.M. Eleiche, J.D. Campbell, Experimental Mechanics, 16, 281 (1976)

5. A. Thakur, S. NematNasser, K.S. Vecchio, Acta Materialia, 44, 2797 (1996)

6. U.S. Lindholm, Journal of the Mechanics and Physics of Solids, 12, 317 (1964)

7. F. Coghe, L. Rabet, L. Kestens, J. Physique IV, 134, 845 (2006)

8. J. Peirs, P. Verleysen, W. Van Paepegem, J. Degrieck, Proceedings Photomechanics2011 (2011)

9. P. Lava, S. Cooreman, M. De Strycker and D. Debruyne, Optics and Lasers in Engineering, 47, 747 (2009)

10. J. Peirs, P. Verleysen, W. Van Paepegem, J. Degrieck, Int. Journal of Impace Engineering, 35, 406 (2011) 